\title{
Article \\ High-Precision and High-Resolution Synchrosqueezing Transform via Time-Frequency Instantaneous Phases
}

\author{
Yong $\mathrm{Li}^{1,2,3}$ and Gulan Zhang ${ }^{3,4, *}$ \\ 1 State Key Laboratory of Shale Oil and Gas Enrichment Mechanisms and Effective Development, \\ Beijing 100083, China; yli0620@163.com \\ 2 SinoPEC Key Laboratory of Seismic Elastic Wave Technology, Beijing 100083, China \\ 3 School of Geoscience and Technology, Southwest Petroleum University, Chengdu 610500, China \\ 4 State Key Laboratory of Oil and Gas Reservoir Geology and Exploitation, Chengdu 610500, China \\ * Correspondence: gulanzhang@163.com
}

check for updates

Citation: Li, Y.; Zhang, G.

High-Precision and High-Resolution Synchrosqueezing Transform via Time-Frequency Instantaneous Phases. Appl. Sci. 2021, 11, 11760. https://doi.org/10.3390/ app112411760

Academic Editor: Atsushi Mase

Received: 21 November 2021

Accepted: 7 December 2021

Published: 10 December 2021

Publisher's Note: MDPI stays neutral with regard to jurisdictional claims in published maps and institutional affiliations.

Copyright: (c) 2021 by the authors. Licensee MDPI, Basel, Switzerland. This article is an open access article distributed under the terms and conditions of the Creative Commons Attribution (CC BY) license (https:// creativecommons.org/licenses/by/ $4.0 /)$.

\begin{abstract}
Synchrosqueezing transform (SST) can effectively improve time-frequency precision and resolution by squeezing time-frequency spectra via instantaneous frequencies, and it has been applied in many diverse disciplines; however, the precision of estimated instantaneous frequencies during SST is usually affected by the time-sample interval of the inputted signal; this usually leads to low-precision or inaccurate SST results and limits its further application. To obtain high-precision and high-resolution SST results with high efficiency, we propose a high-precision and high-resolution SST via time-frequency instantaneous phases (HSST); in HSST, time-frequency instantaneous phases with period-jumps removal are used for high-precision instantaneous frequencies estimation and SST. Two synthetic signal examples show that HSST can minimize the impact of the time-sample interval to achieve high-precision and high-resolution SST results with high efficiency. A real 3D seismic data application demonstrates that HSST has fantastic performance in time-frequency precision and resolution enhancement, and it can be widely used in digital signals processing and interpretation fields.
\end{abstract}

Keywords: synchrosqueezing transform; instantaneous frequency; time-sample interval; instantaneous phase; period-jumps; high-precision; high-resolution

\section{Introduction}

The classical time-frequency methods, such as short-time Fourier transform (STFT) [1], wavelet transform [2,3], S transform [4,5], generalized S transform [6], modified short-time Fourier transform (MSTFT) [7-9], synchrosqueezing transform (SST) [10-21], and so on, have been developed into a mature set of techniques for non-linear and non-stationary digital signals analysis, processing, and interpretation.

Compared with these classical time-frequency methods [1-9], SST can effectively improve time-frequency precision and resolution by time-frequency spectra reassignment via instantaneous frequencies, and this makes SST more suitable for non-linear and nonstationary signals. However, precisions of estimated instantaneous frequencies during SST are decreased with the increasing time-sample interval of the inputted signal; this usually leads to low-precision or inaccurate SST results $[15,18,19,21]$ and limits its further application.

To obtain high-precision and high-resolution SST results, Oberlin et al. proposed a second-order SST via STFT [15], Huang et al. proposed a second-order SST via S transform [18], Pham and Meignen proposed a high-order SST via STFT [19]. In second-order SST, instantaneous phases of the inputted signal are considered second-order functions of time and expressed by second-order Taylor expansion, and second-order partial derivatives of time-frequency spectra with respect to time and frequency are used for instantaneous frequency estimation; whereas in high-order SST, instantaneous phases are considered high-order functions of time and expressed by high-order Taylor expansion, and high-order 
partial derivatives of time-frequency spectra with respect to time and frequency are used for instantaneous frequency estimation. The second or high-order functions cannot well match complex instantaneous phases or complex signals, and second or high-order SST has low processing efficiency due to multiple times partial derivative operations. In order to minimize the impact of the time-sample interval to obtain desirable high-precision and high-resolution SST results, Zhang et al. proposed an adaptive resampled high-resolution SST [21], which is referred to as ASST. In ASST, the resampled time-sample interval is inversely proportional to the highest frequency in the effective frequency band $[9,21]$ of the inputted signal, it can minimize the impact of time-sample interval to obtain high-precision and high-resolution SST results but at the expense of processing efficiency.

In this paper, we propose a high-efficiency, high-precision, and high-resolution SST via time-frequency instantaneous phases (instantaneous phases [22,23] of time-frequency spectra) with period-jumps removal, which is referred to as HSST; in HSST, time-frequency instantaneous phases with period-jumps removal are used for high-precision instantaneous frequencies estimation and SST. Synthetic signals and real 3D seismic data examples demonstrate that HSST can be widely used for seismic signal $[8,9,13,16]$, microseismic signal [24,25], gear vibration signal [6], gravitational-wave signal [19], and other nonstationary digital signals processing and interpretation.

\section{Principles and Methods}

\subsection{Conventional SST (CSST)}

If complex numbers $G(t, f)$ denote the time-frequency spectra of the inputted signal $g(t)$, then SST results can be expressed as

$$
S_{1}(t, v)=\int G(t, f) d f, \text { if } \quad V_{1}(t, f) \in[v-\Delta v, v+\Delta v]
$$

and

$$
V_{1}(t, f)=\operatorname{Re}\left[\frac{1}{2 \pi i} \frac{1}{G(t, f)} \frac{\partial G(t, f)}{\partial t}\right]
$$

where $v$ and $f$ are both frequencies; $\Delta v$ is the frequency increment; $\operatorname{Re}[]$ takes the real part of a complex number; $V_{1}(t, f)$ denote the instantaneous frequencies of the timefrequency spectra $G(t, f)$, which are referred to as time-frequency instantaneous frequencies; $i=\sqrt{-1}$. If

$$
\left\{\begin{array}{l}
x(t, f)=\operatorname{Re}[G(t, f)] \\
y(t, f)=\operatorname{Im}[G(t, f)] \\
G(t, f)=x(t, f)+i y(t, f)
\end{array}\right.
$$

where $\operatorname{Im}[$ ] takes an imaginary part of a complex number, respectively, then

$$
V_{1}(t, f)=\frac{1}{2 \pi} \frac{x(t, f) \frac{\partial y(t, f)}{\partial t}-y(t, f) \frac{\partial x(t, f)}{\partial t}}{x^{2}(t, f)+y^{2}(t, f)} .
$$

For actual discrete signals, Equation (4) should be modified as (see Appendix A)

$$
V_{2}(t, f)=\frac{1}{2 \pi \Delta t} \frac{x(t-\Delta t, f) y(t, f)-y(t-\Delta t, f) x(t, f)}{\left[x^{2}(t, f)+y^{2}(t, f)\right]}
$$

where $\Delta t$ is the time-sample interval of the inputted signal $g(t)$; and $V_{2}(t, f)$ can be considered the estimated time-frequency instantaneous frequencies of $G(t, f)$; correspondingly, Equation (1) can be rewritten as

$$
S_{2}(t, v)=\int G(t, f) d f, \text { if } \quad V_{2}(t, f) \in[v-\Delta v, v+\Delta v]
$$

which is considered conventional SST (CSST). 
Precisions of $V_{2}(t, f)$ are inversely proportional to the time-sample interval, this usually leads to inaccurate SST results [21]. To minimize the impact of the time-sample interval, Zhang et al. proposed ASST [21], which can minimize the impact of the timesample interval to obtain high-precision and high-resolution SST results, but at the expense of processing efficiency.

Figure 1a-c show time-frequency instantaneous amplitudes (modulus of time-frequency spectra) of a $50 \mathrm{~Hz}$ harmonic signal obtained by MSTFT, CSST, and ASST with $\Delta t=2$ milliseconds (ms), respectively; Figure $1 \mathrm{~d}-\mathrm{f}$ show time-frequency instantaneous amplitudes of the $50 \mathrm{~Hz}$ harmonic signal obtained by MSTFT, CSST, and ASST with $\Delta t=4 \mathrm{~ms}$, respectively; Table 1 shows the time consuming of MSTFT, CSST, and ASST for the $50 \mathrm{~Hz}$ harmonic signal with $4 \mathrm{~s}$ length. The peak frequencies in Figure $1 \mathrm{a}-\mathrm{f}$ are 50, 48, 50, 50, 37, and $50 \mathrm{~Hz}$, respectively; we can see that CSST obtains incorrect SST results, and ASST obtains higher precision and resolution results than MSTFT but with lower processing efficiency.

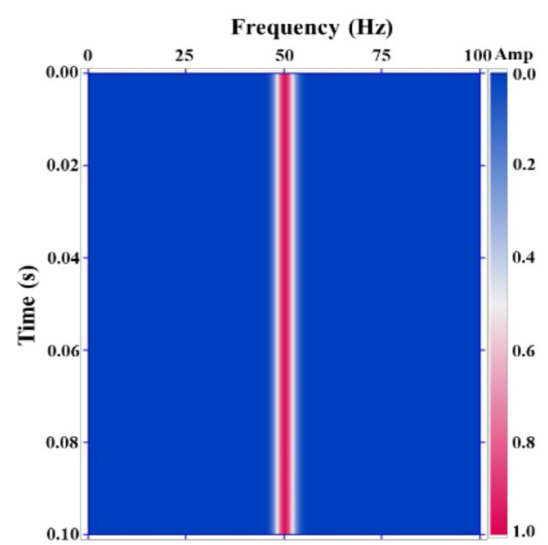

(a)

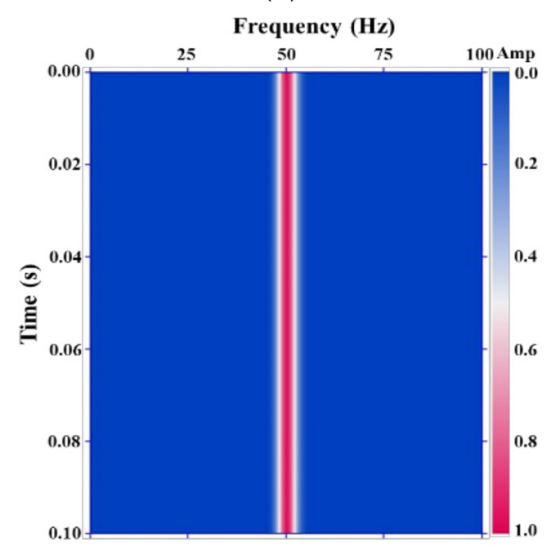

(d)

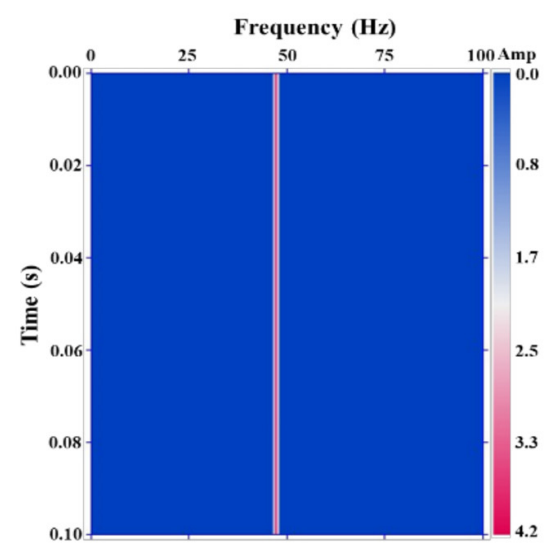

(b)

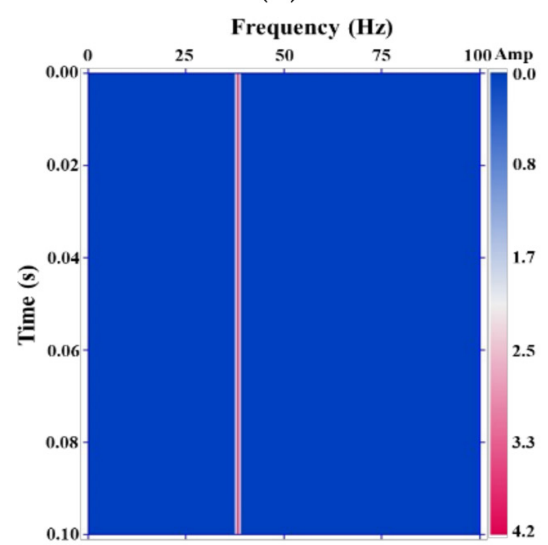

(e)

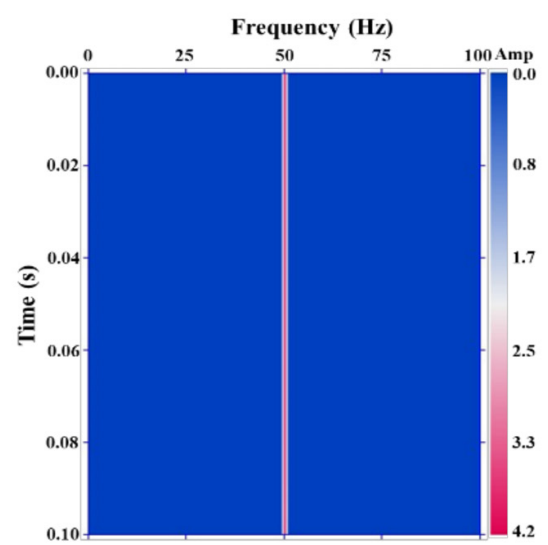

(c)

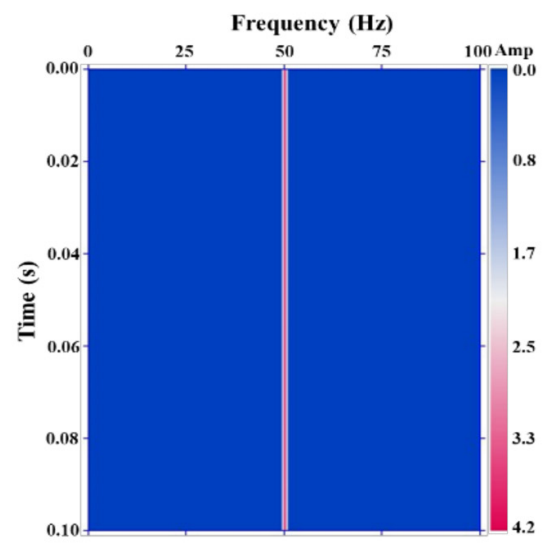

(f)

Figure 1. Time-frequency instantaneous amplitudes of a $50 \mathrm{~Hz}$ harmonic signal obtained by MSTFT with (a) $\Delta t=2 \mathrm{~ms}$ and (d) $\Delta t=4 \mathrm{~ms}$; CSST with (b) $\Delta t=2 \mathrm{~ms}$ and (e) $\Delta t=4 \mathrm{~ms}$; ASST with (c) $\Delta t=2 \mathrm{~ms}$ and (f) $\Delta t=4 \mathrm{~ms}$.

Table 1. Time consuming of MSTFT, CSST, and ASST for the $50 \mathrm{~Hz}$ harmonic signal with $4 \mathrm{~s}$ length.

\begin{tabular}{|c|c|c|c|c|c|c|}
\hline \multirow{2}{*}{$\begin{array}{c}\text { Time-Sample Intervals } \\
\text { Methods }\end{array}$} & \multicolumn{3}{|c|}{$\Delta t=2 \mathrm{~ms}$} & \multicolumn{3}{|c|}{$\Delta t=4 \mathrm{~ms}$} \\
\hline & MSTFT & CSST & ASST & MSTFT & CSST & ASST \\
\hline Time consuming (s) & $\mathrm{M} 1=16.0$ & $\mathrm{C} 1=16.2$ & $\mathrm{~A} 1=33.2$ & $\mathrm{M} 2=9.7$ & $\mathrm{C} 2=9.8$ & $\mathrm{~A} 2=20.1$ \\
\hline Ratios & \multicolumn{3}{|c|}{$\mathrm{A} 1 / \mathrm{M} 1=2.08 ; \mathrm{A} 1 / \mathrm{C} 1=2.05$} & \multicolumn{3}{|c|}{$\mathrm{A} 2 / \mathrm{M} 2=2.07 ; \mathrm{A} 2 / \mathrm{C} 2=2.05$} \\
\hline
\end{tabular}


2.2. High-Precision and High-Resolution SST via Time-Frequency Instantaneous Phases (HSST)

According to Appendix B, we can use instantaneous phases of $G(t, f)$ for highprecision time-frequency instantaneous frequency estimation and finally obtain highprecision and high-resolution SST results with high efficiency.

If $\theta(t, f)$ and $\theta(t, f) \in(-\infty,+\infty)$ denote the argument angles of $G(t, f), \theta_{1}(t, f)$ and $\theta_{1}(t, f) \in[0,2 \pi]$ denote the principal argument angles of $G(t, f)$, then we have

$$
\left\{\begin{array}{l}
\theta(t, f)=2 k \pi+\theta_{1}(t, f) \\
\theta\left(t_{2}, f\right)>\theta\left(t_{1}, f\right), \text { if } t_{2}>t_{1}
\end{array}\right.
$$

where $k$ denote integer numbers; $\theta(t, f)$ can be considered time-frequency instantaneous phases of $G(t, f) ; \theta_{1}(t, f)$ can be considered time-frequency principal instantaneous phases of $G(t, f)$ given by

$$
\theta_{1}(t, f)=\left\{\begin{array}{c}
0.5 \pi, \text { if } x(t, f)=0, y(t, f) \geq 0 \\
1.5 \pi, \text { if } x(t, f)=0, y(t, f)<0 \\
\theta_{0}(t, f), \text { if } x(t, f)>0, y(t, f) \geq 0 \\
\pi+\theta_{0}(t, f), \text { if } x(t, f)<0, y(t, f) \geq 0 \\
\pi+\theta_{0}(t, f), \text { if } x(t, f)<0, y(t, f)<0 \\
2 \pi+\theta_{0}(t, f), \text { if } x(t, f)>0, y(t, f)<0
\end{array}\right.
$$

and

$$
\theta_{0}(t, f)=\arctan \left[x^{-1}(t, f) \cdot y(t, f)\right] \in(-0.5 \pi, 0.5 \pi)
$$

According to Equations (A6) and (A16), the time-frequency instantaneous frequencies of $G(t, f)$ can be given by [22]

$$
V_{1}(t, f)=\frac{1}{2 \pi} \frac{\partial \theta(t, f)}{\partial t}=\frac{1}{2 \pi} \lim _{\Delta t \rightarrow 0}\left[\frac{\theta(t, f)-\theta(t-\Delta t, f)}{\Delta t}\right]
$$

but parameter $k$ in Equation (7) cannot be determined; thus, $\theta(t, f)$ cannot be used for time-frequency instantaneous frequencies estimation.

If $\theta_{0}(t, f)$ are used for time-frequency instantaneous frequencies estimation, according to Equations (A16)-(A19), $V_{1}(t, f)$ in Equation (10) can be obtained by computing the derivative of the arctangent function itself to avoid period-jumps of $\theta_{0}(t, f)$ as

$$
V_{3}(t, f)=\frac{1}{2 \pi} \frac{\partial \theta_{0}(t, f)}{\partial t}=\frac{1}{2 \pi} \frac{\partial \arctan \left[x^{-1}(t, f) \cdot y(t, f)\right]}{\partial t}
$$

and

$$
V_{3}(t, f)=\frac{1}{2 \pi} \frac{x(t, f) \frac{\partial y(t, f)}{\partial t}-y(t, f) \frac{\partial x(t, f)}{\partial t}}{x^{2}(t, f)+y^{2}(t, f)}=V_{1}(t, f) .
$$

Then, the estimated time-frequency instantaneous frequencies can be given by

$$
V_{4}(t, f)=\frac{1}{2 \pi \Delta t} \frac{x(t-\Delta t, f) y(t, f)-y(t-\Delta t, f) x(t, f)}{x^{2}(t, f)+y^{2}(t, f)}=V_{2}(t, f)
$$

Thus, according to [21] and Figure 1, it avoids period-jumps of $\theta_{0}(t, f)$, but it cannot avoid the impact of the time-sample interval.

If $\theta_{1}(t, f)$ is used for instantaneous frequencies estimation, then according to Equation (A21), the estimated time-frequency instantaneous frequencies can be given by

$$
V_{5}(t, f)=\frac{1}{2 \pi} \frac{\theta_{1}(t, f)-\theta_{1}(t-\Delta t, f)}{\Delta t}=\frac{1}{2 \pi} \frac{\Delta \theta_{1}(t, f)}{\Delta t}
$$


and the corresponding SST results can be expressed as

$$
S_{3}(t, v)=\int G(t, f) d f, \text { if } \quad V_{5}(t, f) \in[v-\Delta v, v+\Delta v]
$$

which is considered SST via the time-frequency principal instantaneous phases (PSST); PSST can avoid the impact of the time-sample interval to obtain high-resolution SST results, except for time-points corresponding to period-jumps of $\theta_{1}(t, f)$.

According to Equations (A21)-(A27), if we considered

$$
\Delta \theta_{2}(t, f)=\left\{\begin{array}{l}
-\Delta \theta_{1}(t, f)-2 \pi, \text { if } \quad-2 \pi \leq \Delta \theta_{1}(t, f)<-\pi \\
\Delta \theta_{1}(t, f), \text { if }-\pi \leq \Delta \theta_{1}(t, f) \leq \pi \\
\Delta \theta_{1}(t, f)-2 \pi, \quad \text { if } \pi<\Delta \theta_{1}(t, f) \leq 2 \pi
\end{array}\right.
$$

then, estimated high-precision time-frequency instantaneous frequencies can be given by

$$
V_{6}(t, f)=\frac{1}{2 \pi} \frac{\Delta \theta_{2}(t, f)}{\Delta t}
$$

and corresponding SST results can be expressed as

$$
S_{4}(t, v)=\int G(t, f) d f, \text { if } \quad V_{6}(t, f) \in[v-\Delta v, v+\Delta v]
$$

which is considered HSST; HSST can avoid the impacts of the time-sample interval and period-jumps of $\theta_{1}(t, f)$ to obtain high-precision and high-resolution SST results with high-efficiency.

Figure 2a shows the time-frequency principal instantaneous phases of the $50 \mathrm{~Hz}$ harmonic signal; Figure $2 b, c$ show the time-frequency instantaneous amplitudes obtained by PSST with $\Delta t=2$ and $4 \mathrm{~ms}$, respectively; Figure $2 \mathrm{~d}$, e show the time-frequency instantaneous amplitudes obtained by HSST with $\Delta t=2$ and $4 \mathrm{~ms}$, respectively. Table 2 shows the time consuming of PSST and HSST for the $50 \mathrm{~Hz}$ harmonic signal with $4 \mathrm{~s}$ length.

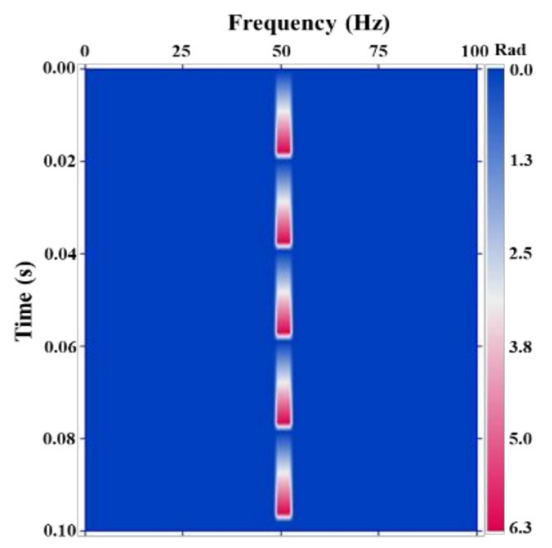

(a)

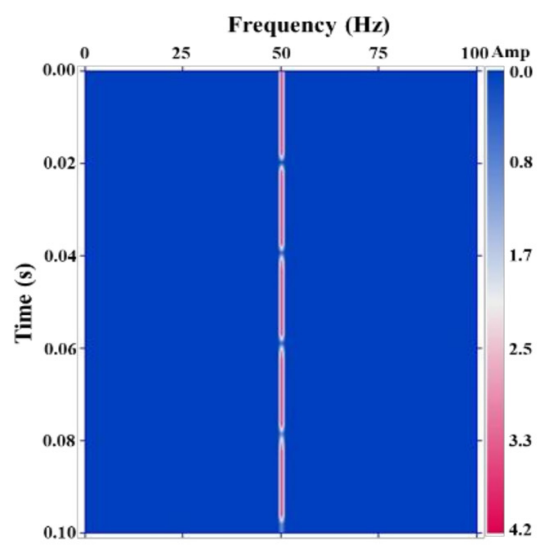

(b)

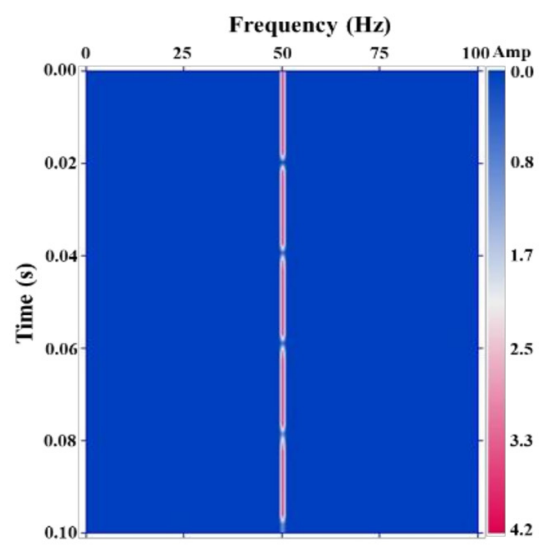

(c)

Figure 2. Cont. 


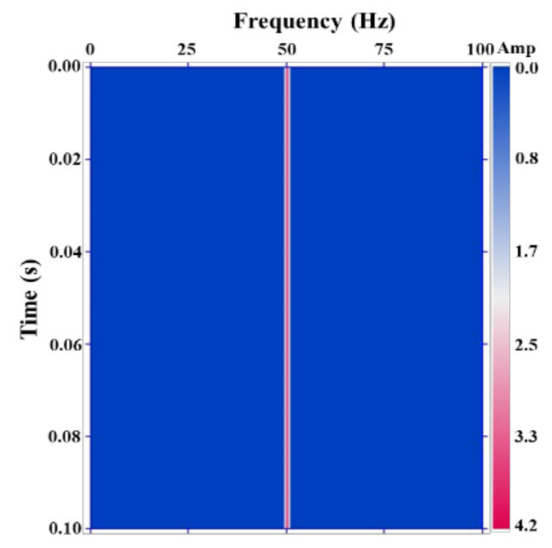

(d)

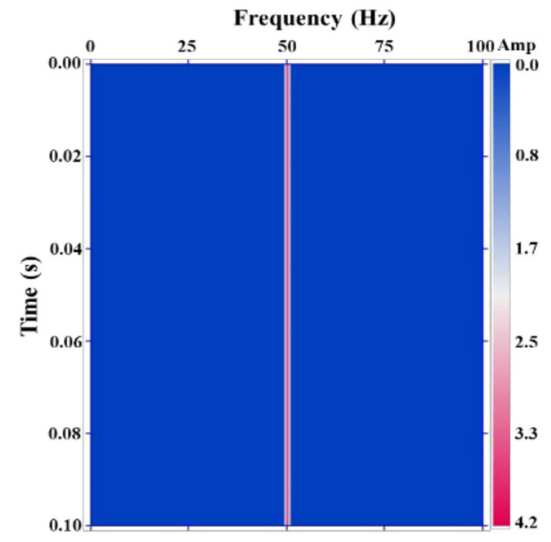

(e)

Figure 2. $50 \mathrm{~Hz}$ harmonic signal: (a) Time-frequency principal instantaneous phases; Time-frequency instantaneous amplitudes obtained by PSST with (b) $\Delta t=2 \mathrm{~ms}$ and (c) $\Delta t=4 \mathrm{~ms}$. Time-frequency instantaneous amplitudes obtained by HSST with (d) $\Delta t=2 \mathrm{~ms}$ and (e) $\Delta t=4 \mathrm{~ms}$.

Table 2. Time-consuming of PSST and HSST for the $50 \mathrm{~Hz}$ harmonic signal with $4 \mathrm{~s}$ length.

\begin{tabular}{ccccc}
\hline Time-Sample Intervals & \multicolumn{2}{c}{$\Delta \boldsymbol{t}=\mathbf{2} \mathbf{~ m s}$} & \multicolumn{2}{c}{$\Delta \boldsymbol{t}=\mathbf{4} \mathbf{~ m s}$} \\
\hline Methods & PSST & HSST & PSST & HSST \\
\hline Time consuming (s) & $\mathrm{P} 1=16.282$ & $\mathrm{H} 1=16.288$ & $\mathrm{P} 2=9.868$ & $\mathrm{H} 2=9.872$ \\
Ratios & $\mathrm{H} 1 / \mathrm{M} 1=1.019 ; \mathrm{H} 1 / \mathrm{C} 1=1.004$ & $\mathrm{H} 2 / \mathrm{M} 2=1.019 ; \mathrm{H} 2 / \mathrm{C} 2=1.004$ \\
\hline
\end{tabular}

Comparison of Figures 1 and 2 shows that PSST has higher precision and resolution than MSTFT and CSST, except for time-points corresponding to period-jumps of timefrequency principal instantaneous phases; HSST avoids period-jumps during PSST, and it has higher precision and resolution than MSTFT, CSST, and PSST.

\section{Examples}

In this section, a synthetic signal and 3D real seismic data are used to demonstrate the high-precision and high-resolution abilities of HSST with comparisons of CSST, ASST, and PSST; and the time-frequency spectra for CSST, ASST, PSST, and HSST are obtained by MSTFT [7] given by

$$
G(t, f)=\int_{-\infty}^{+\infty} z(\tau+t) w(\tau) \exp (-i 2 \pi f \tau) d \tau
$$

and

$$
w(\tau)=\frac{a|f|^{b}}{\sqrt{2 \pi}} \exp \left[-\frac{a^{2}|f|^{2 b}}{2} \tau^{2}\right]
$$

where $z(t)$ is the analytic signal [22] of the inputted signal $g(t) ; a>0$ and $b \geq 0$.

\subsection{Synthetic Signal Example}

We consider a synthetic signal as

$$
g(t)=\cos (180 \pi t+30 \times \sin 4 \pi t)
$$

and its instantaneous frequencies can be expressed as

$$
f(t)=90+60 \times \cos 4 \pi t \text {. }
$$


Figure $3 a, b$ show the synthetic signal and its instantaneous frequencies, respectively. Figure 4a-e show time-frequency instantaneous amplitudes obtained by MSTFT, CSST, ASST, PSST, and HSST with $\Delta t=1 \mathrm{~ms}$, respectively; Figure $4 \mathrm{f}$ shows the differences between Figure 4 d,e. We can see that the peak frequencies in Figure $4 a, c, e$ are almost equal to the instantaneous frequencies shown in Figure $3 b$, whereas the peak frequencies in Figure $4 \mathrm{~b}$ show some deviations from the instantaneous frequencies shown in Figure $3 \mathrm{~b}$, and these deviations become more obvious with the increasing instantaneous frequencies. A comparison of Figure 4a-c,e shows that Figure 4c,e are almost the same and both have higher precisions and resolutions than Figure 4a; Figure $4 \mathrm{~b}$ obtains incorrect SST results.

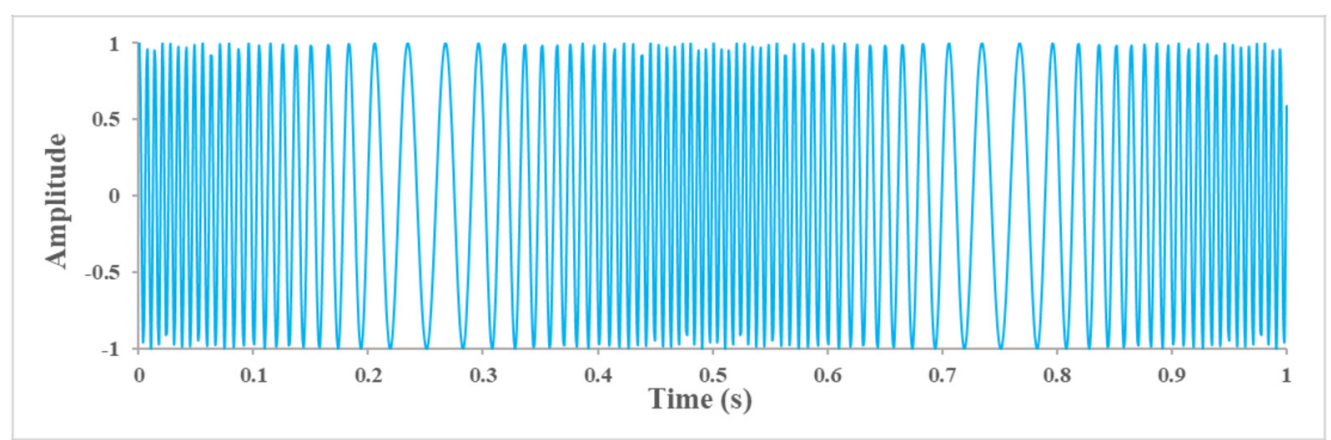

(a)

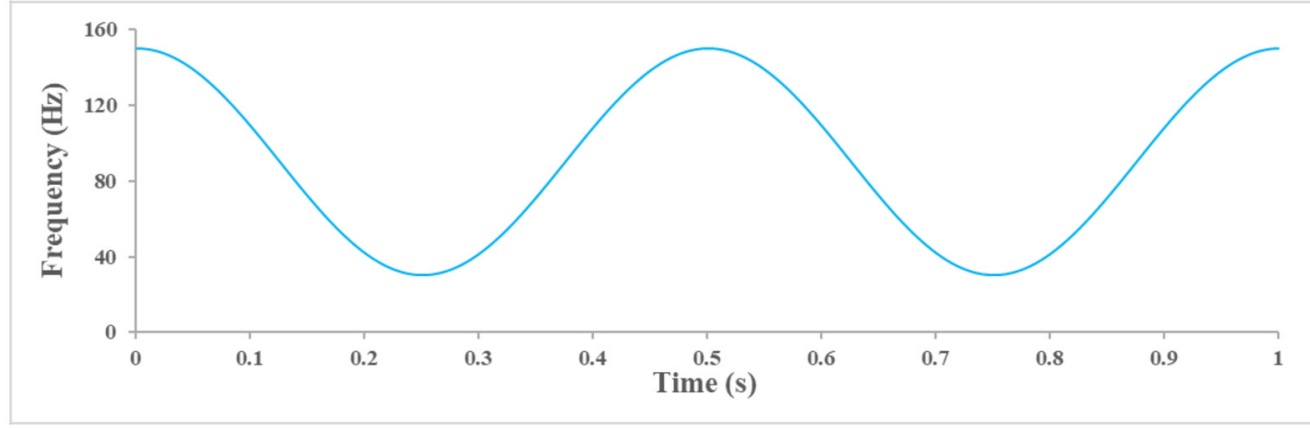

(b)

Figure 3. (a) Synthetic signal; (b) Instantaneous frequencies.

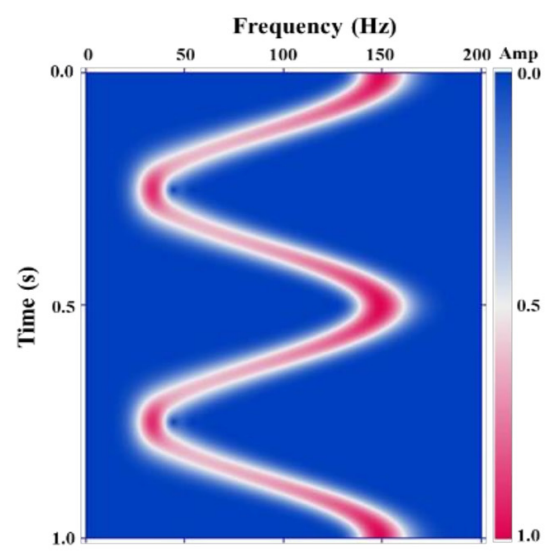

(a)

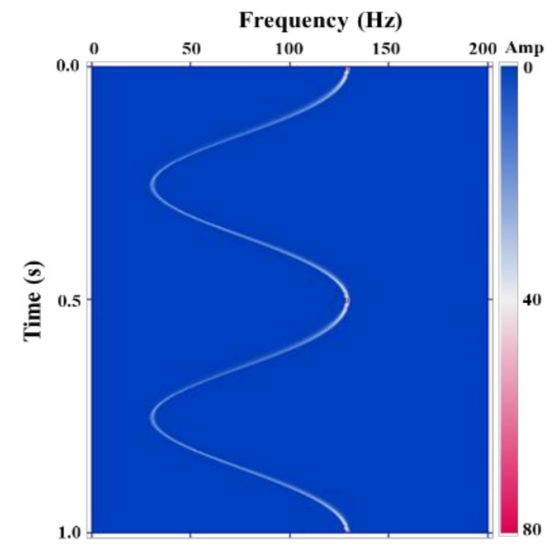

(b)

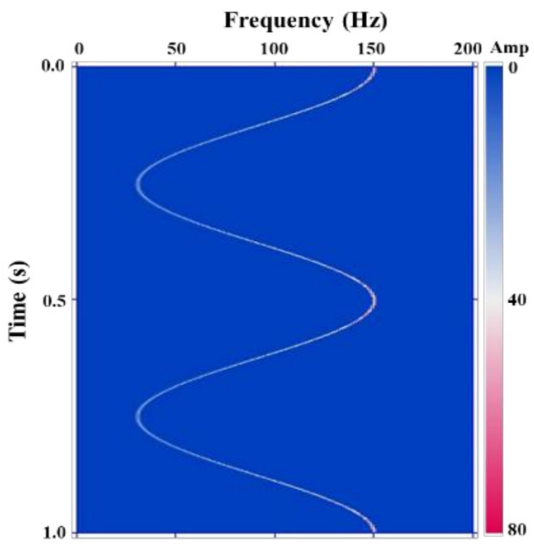

(c)

Figure 4. Cont. 


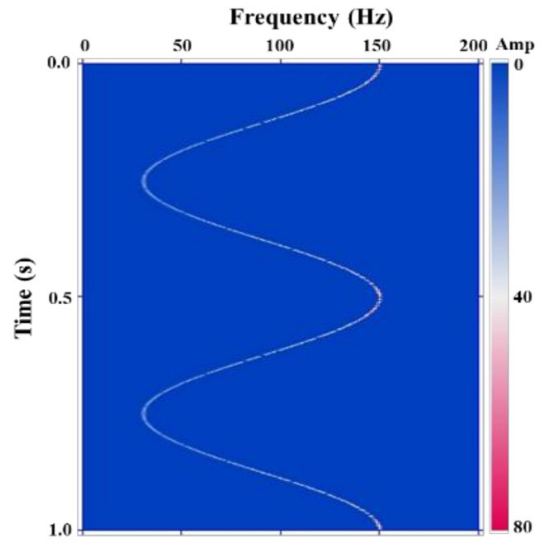

(d)

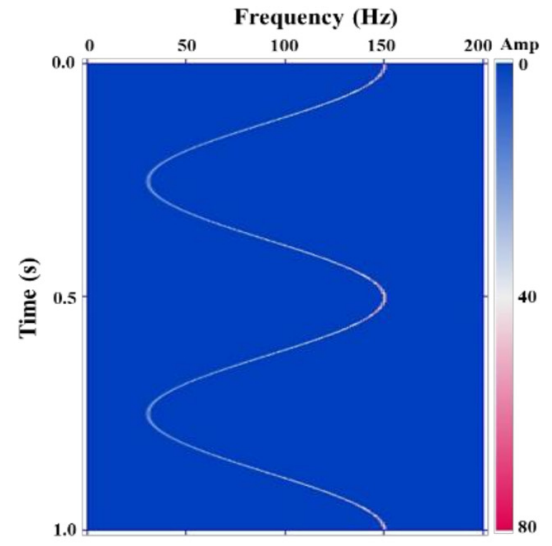

(e)

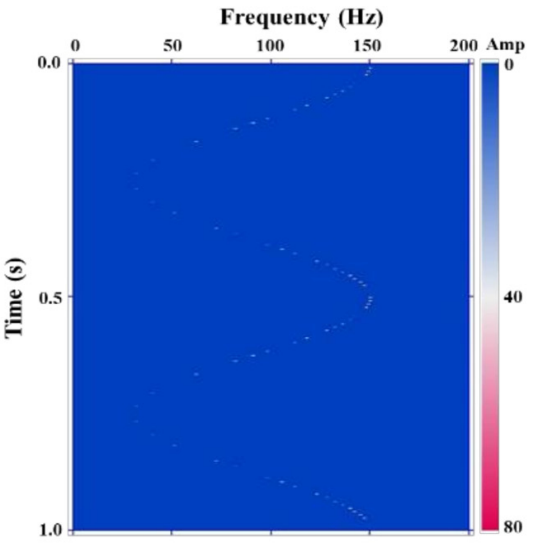

(f)

Figure 4. Time-frequency instantaneous amplitudes obtained by (a) MSTFT; (b) CSST; (c) ASST; (d) PSST; (e) HSST; (f) Differences between $(\mathbf{d}, \mathbf{e})$.

Figure $4 \mathrm{~d}$ has the same results as Figure 4e, except for time-points corresponding to period-jumps of time-frequency principal instantaneous phases; thus, Figure 4e has higher precision and better continuity than Figure 4d. Above all, HSST has higher precision and resolution than MSTFT, CSST, and PSST, and almost the same results as ASST.

\subsection{D Field Seismic Data Example}

We applied MSTF, CSST, PSST, and HSST to a 3D field seismic data with $\Delta t=2 \mathrm{~ms}$.

Figure 5a-c show amplitudes, instantaneous amplitudes, and principal instantaneous phases of crossline 1 extracted from the field 3D seismic data, respectively.

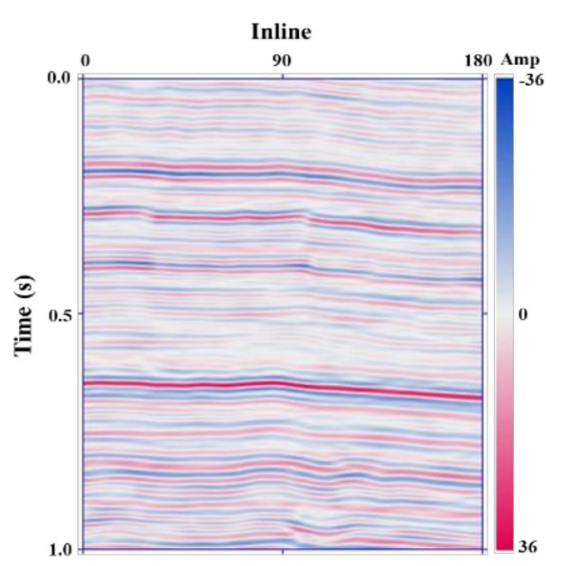

(a)

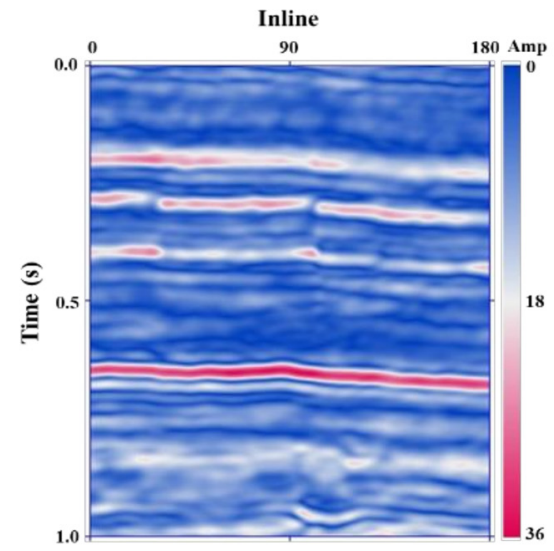

(b)

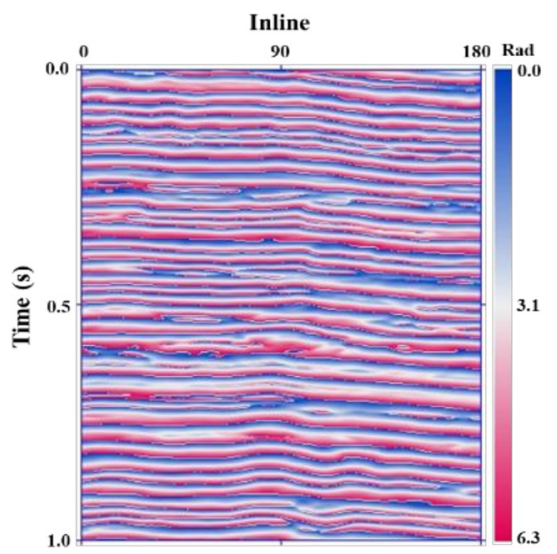

(c)

Figure 5. Crossline 1: (a) Amplitudes; (b) Instantaneous amplitudes; (c) Principal instantaneous phases.

Figure 6a shows time-frequency instantaneous principal instantaneous phases of the 10th trace in Figure 5a, which are obtained by MSTFT; Figure 6b-e show time-frequency instantaneous amplitudes of the 10th trace in Figure 5a obtained by MSTFT, CSST, PSST, and HSST, respectively; Figure $6 \mathrm{f}$ show differences between Figure $6 \mathrm{~d}$,e. Figure $6 \mathrm{~g}-\mathrm{i}$ show zoomed-in views of yellow rectangles in Figure $6 c-e$, respectively. We can see that Figure $6 c-e$ have higher resolutions than Figure 6b, and Figure 6d,e have higher precisions than Figure $6 b, c$; in addition, Figure $6 c$,e have better continuities than Figure $6 d$, as indicated by green arrows. 


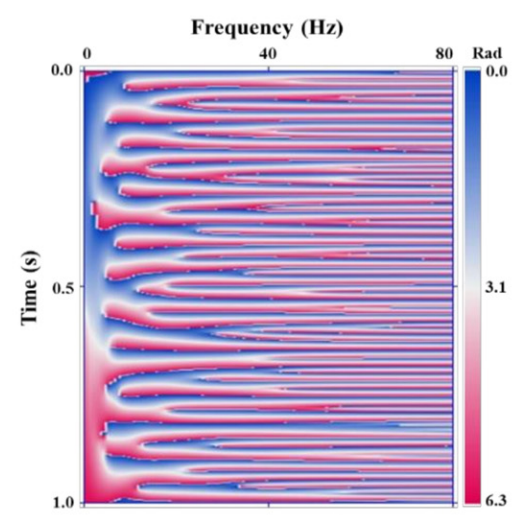

(a)

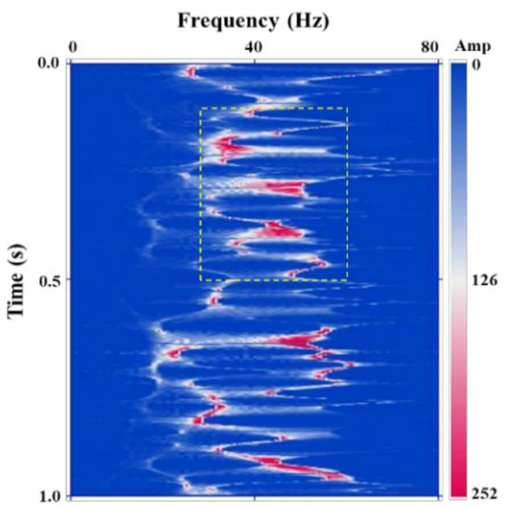

(d)

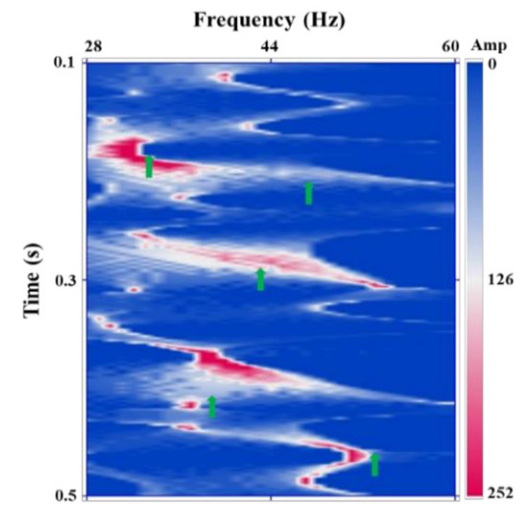

(g)

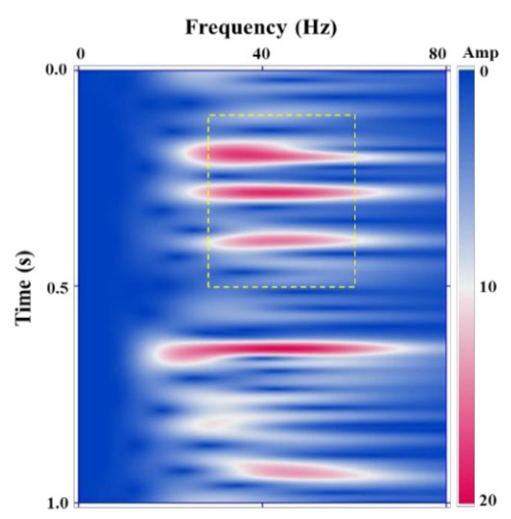

(b)

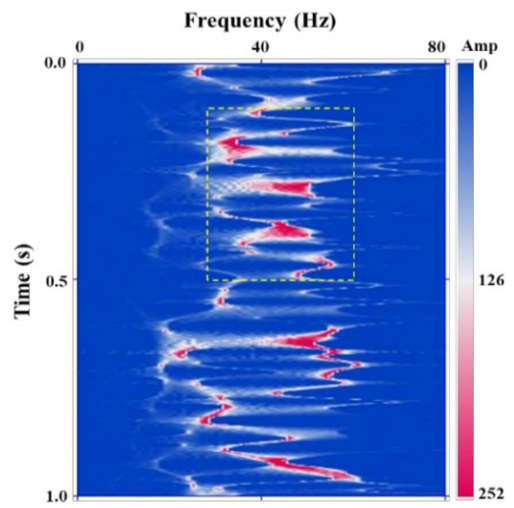

(e)

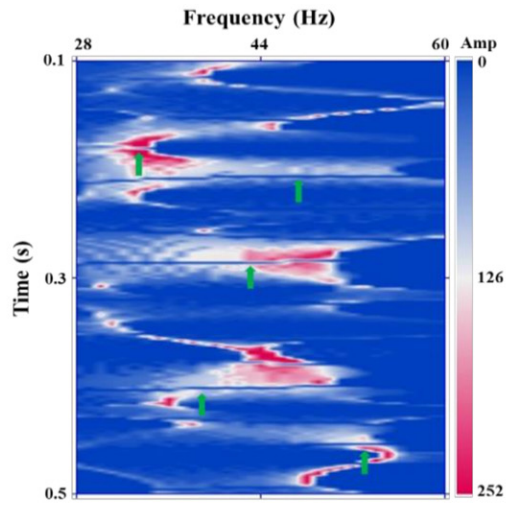

(h)

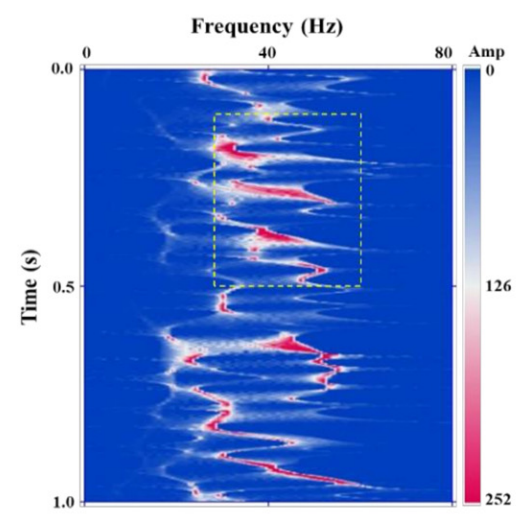

(c)

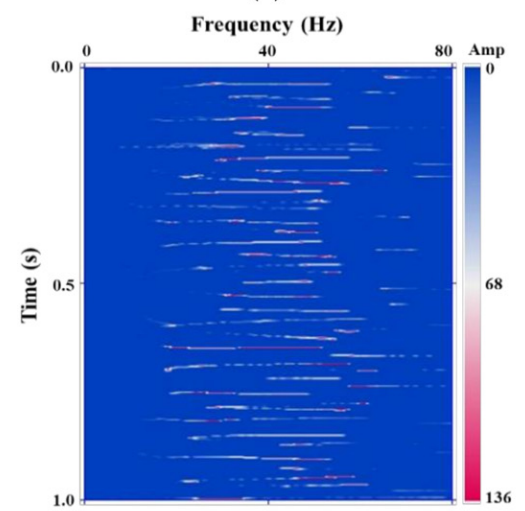

(f)

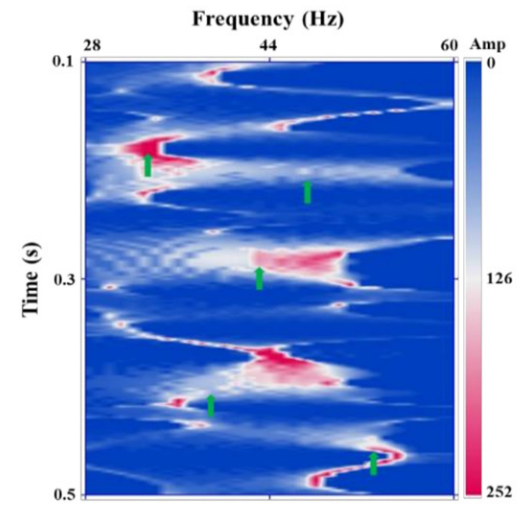

(i)

Figure 6. (a) Time-frequency instantaneous principal instantaneous phases of the 10th trace in Figure 5a obtained by MSTFT; Time-frequency instantaneous amplitudes of the 10th trace in Figure 5a obtained by: (b) MSTFT; (c) CSST; (d) PSST; (e) HSST. (f) Differences between Figure 6d,e; (g) Zoomed-in views of yellow rectangle in Figure 6c; (h) Zoomed-in views of yellow rectangle in Figure 6d; (i) Zoomed-in views of yellow rectangle in Figure 6e.

Figure $7 \mathrm{a}-\mathrm{c}$ show $30 \mathrm{~Hz}$ time-frequency instantaneous amplitudes of crossline 1 obtained by MSTFT, PSST, and HSST, respectively; Figure 7d-f show $50 \mathrm{~Hz}$ time-frequency instantaneous amplitudes of crossline 1 obtained by MSTFT, PSST, and HSST, respectively; Figure $7 \mathrm{~g}$ shows the differences between Figure $7 \mathrm{~b}, \mathrm{c}$, and Figure $7 \mathrm{~h}$ shows the differences between Figure 7e,f. Figure 7b,c have higher resolutions than Figure 7a, and Figure 7e,f have higher resolutions than Figure $7 d$; Figure $7 c$ has higher precision and better continuity than Figure $7 b$, and Figure $7 \mathrm{f}$ has higher precision and better continuity than Figure 7e.

Figure 8a shows a time slice extracted from the field 3D seismic data; Figure 8b,c show the same time slices of instantaneous amplitudes and principal instantaneous phases, 
respectively, obtained by Hilbert transform [22]. As shown in Figure 8b,c, the main channels are represented but with low resolution.

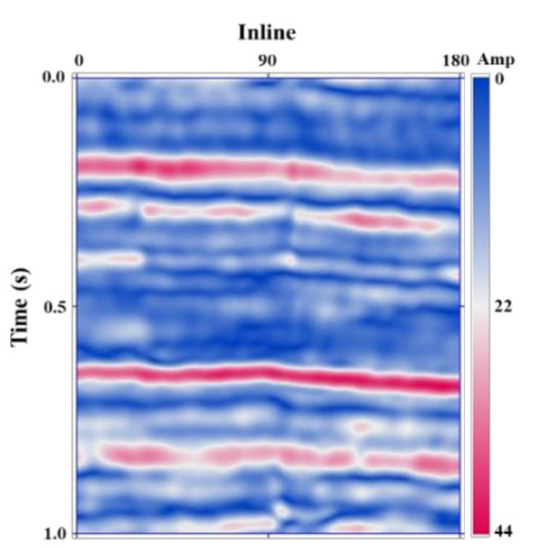

(a)

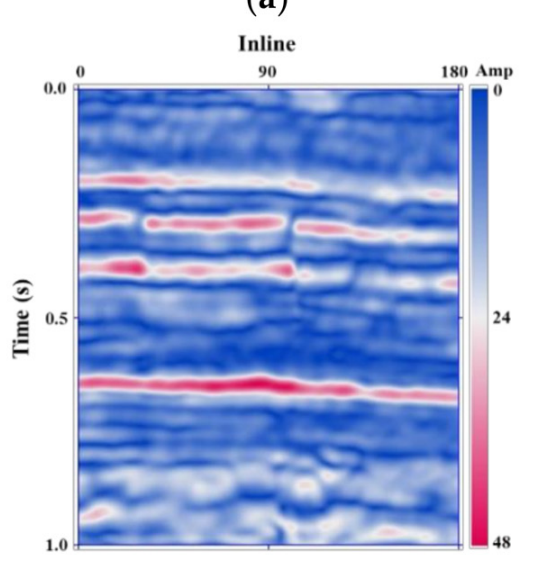

(d)

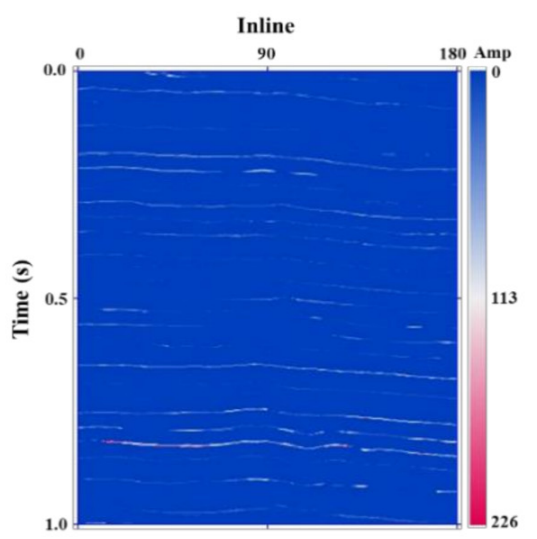

(g)

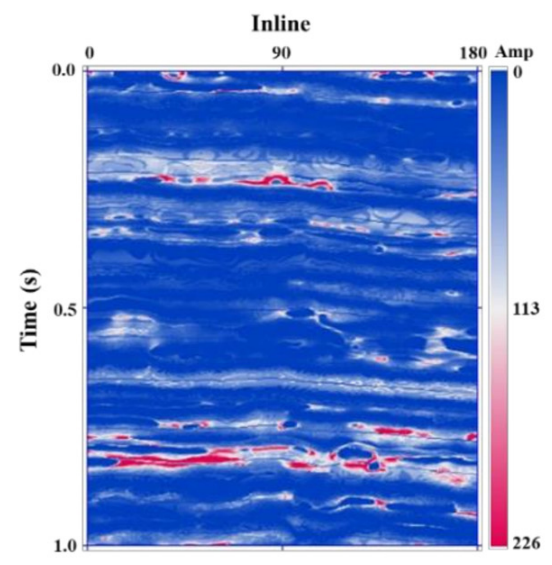

(b)

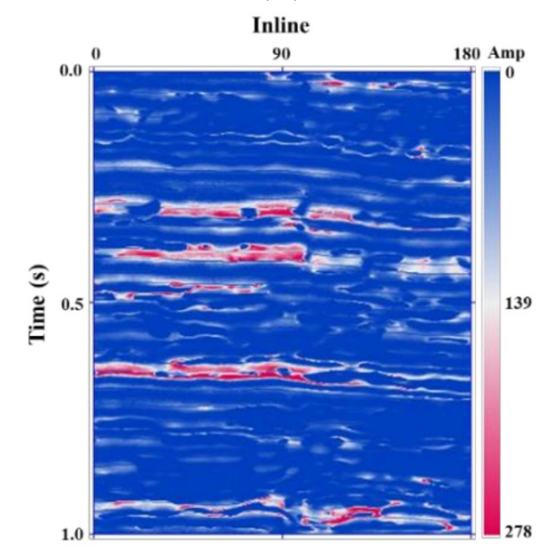

(e)

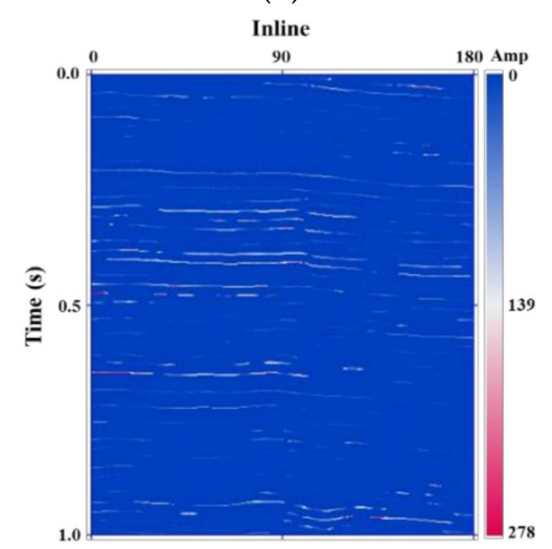

(h)

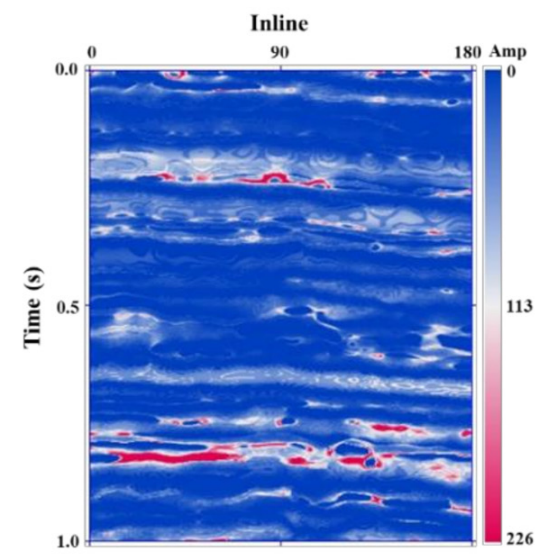

(c)

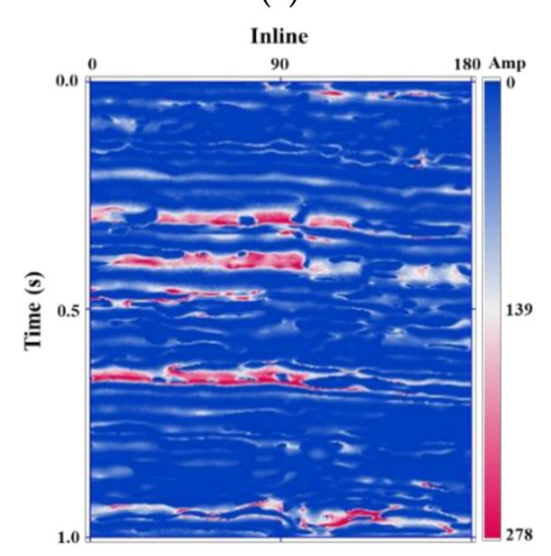

$(\mathbf{f})$

Figure 7. The $30 \mathrm{~Hz}$ time-frequency instantaneous amplitudes of crossline 1 in Figure 5a obtained by (a) MSTFT; (b) PSST; (c) HSST. The $50 \mathrm{~Hz}$ time-frequency instantaneous amplitudes of crossline 1 in Figure 5a obtained by (d) MSTFT; (e) PSST; (f) HSST. (g) Differences between Figure 7b,c; (h) Differences between Figure 7e,f.

Figure $9 a, b, c$ show the same time slices of $30 \mathrm{~Hz}$ time-frequency instantaneous amplitudes obtained by MSTFT, PSST, and HSST, respectively; Figure 9d,e,f show the same time slices of $50 \mathrm{~Hz}$ time-frequency instantaneous amplitudes obtained by MSTFT, PSST, and HSST, respectively; Figure 9g shows the differences between Figure 9b,c, and Figure $9 \mathrm{~h}$ shows the differences between Figure $9 \mathrm{e}, \mathrm{f}$, which are false structures caused by 
period-jumps of time-frequency principal instantaneous phases during PSST. Figure $9 b, c$ have higher resolutions than Figure 9a, and Figure 9e,f have higher resolutions than Figure 9d; in addition, Figure $9 \mathrm{c}$ has higher precisions and better continuities than Figure $9 \mathrm{~b}$, and Figure $9 \mathrm{f}$ has higher precision and better continuity than Figure 9e.

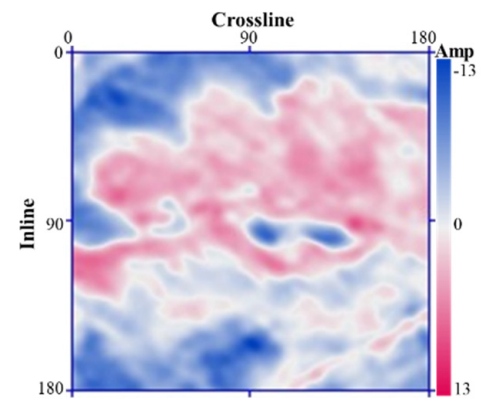

(a)

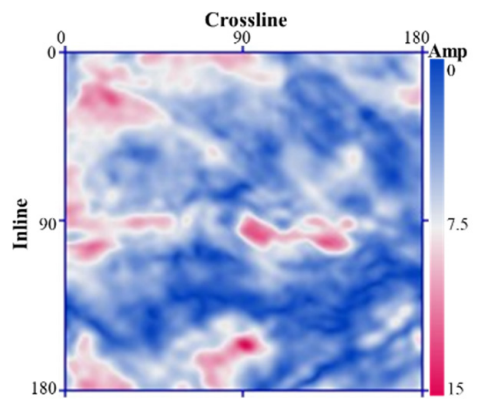

(b)

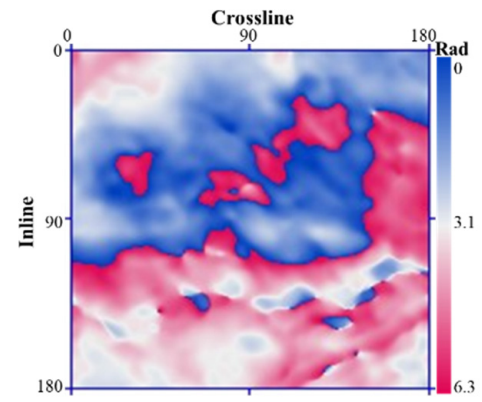

(c)

Figure 8. Time slices: (a) Amplitudes; (b) Instantaneous amplitudes; (c) Principal instantaneous phases.

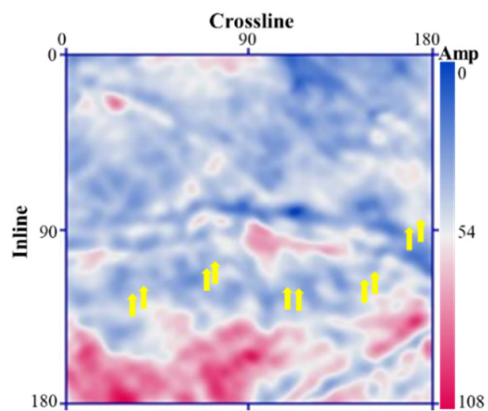

(a)

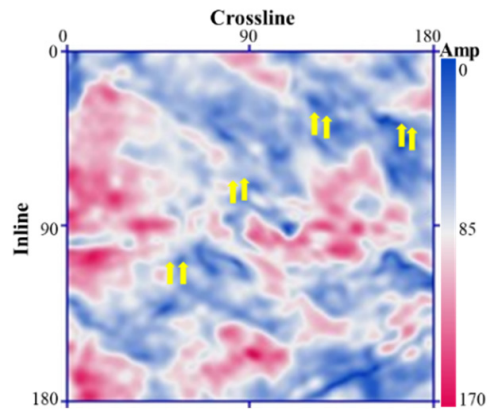

(d)

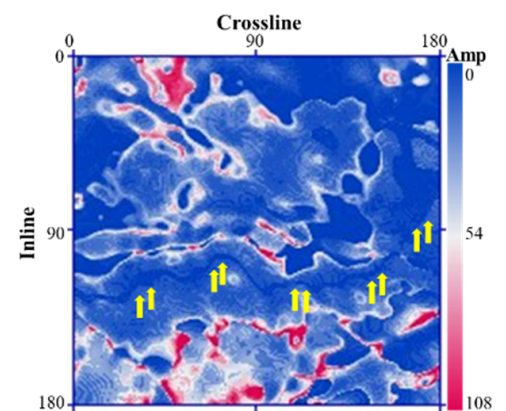

(b)

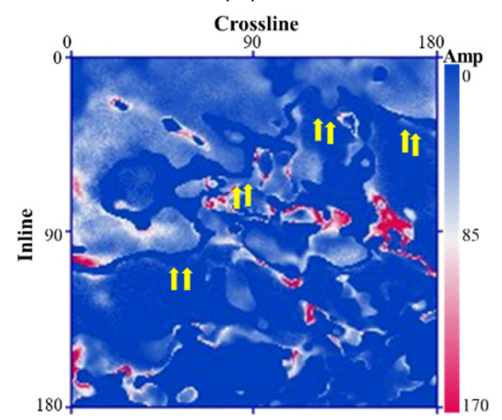

(e)

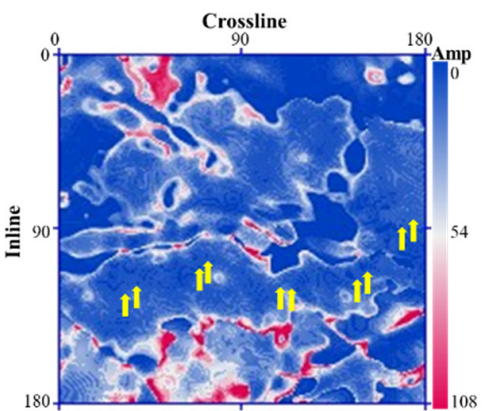

(c)

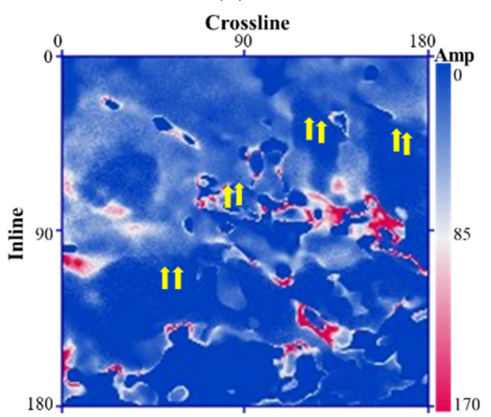

(f)

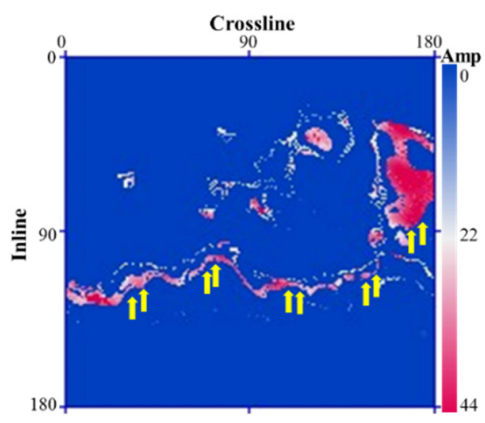

(g)

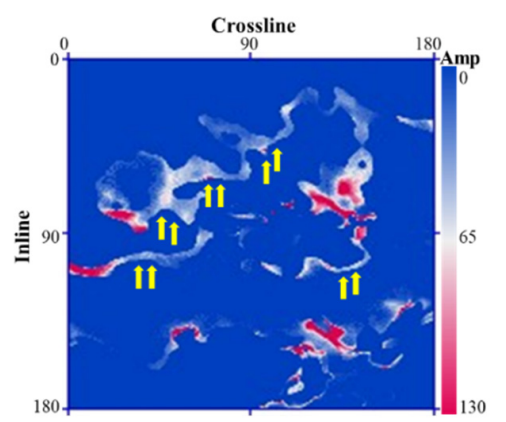

(h)

Figure 9. Time slices of $30 \mathrm{~Hz}$ time-frequency instantaneous amplitudes obtained by (a) MSTFT; (b) PSST; (c) HSST; Time slices of $50 \mathrm{~Hz}$ time-frequency instantaneous amplitudes obtained by (d) MSTFT; (e) PSST; (f) HSST; (g) Differences between Figure 9b,c; (h) Differences between Figure 9e,f. 
Thus, HSST has higher precisions and resolutions than MSTFT, CSST and PSST, and it achieves desirable high-precision and high-resolution SST results.

\section{Conclusions}

To obtain high-precision and high-resolution SST results with high-efficiency, we propose a high-precision and high-resolution SST via time-frequency instantaneous phases, which is referred to as HSST. Compared with CSST, ASST, and PSST, HSST avoids the impact of the time-sample interval during CSST and period-jumps of time-frequency principal instantaneous phases during PSST, and finally, it obtains desirable high-precision and high-resolution SST results with high efficiency. Synthetic signal and field 3D seismic data examples indicate that HSST can be extensively applied in non-linear and non-stationary digital signals analysis, processing, and interpretation fields.

Author Contributions: Writing—original draft preparation, Y.L.; Methodology, G.Z.; Writing—review and editing, G.Z. All authors have read and agreed to the published version of the manuscript.

Funding: The National Natural Science Foundation of China (Grant No. 41874168), the Sichuan Science and Technology Program for Distinguished Young Scholars (Grant No. 2019JDJQ0053), the National Key R\&D Program of China (2018YFA0702505), the National Natural Science Foundation of China- Sinopec Joint Key Project (U19B6003-004), and the National Science and Technology Major Project (2017ZX05005-004).

Institutional Review Board Statement: Not applicable.

Informed Consent Statement: Not applicable.

Conflicts of Interest: The authors declare no conflict of interest.

\section{Appendix A. Conventional Instantaneous Frequencies Estimation Method}

For a complex signal

$$
c(t)=x(t)+i y(t)
$$

where $x(t)$ and $y(t)$ are both real signals; if $A(t)$ and $A(t) \in[0,+\infty)$ denote argument amplitudes of $c(t), \theta(t)$ and $\theta(t) \in(-\infty,+\infty)$ denote argument angles of $c(t), \theta_{1}(t)$ and $\theta_{1}(t) \in[0,2 \pi]$ denote principal argument angles of $c(t)$; then

$$
c(t)=A(t) \exp [i \theta(t)]
$$

and

$$
\left\{\begin{array}{l}
A(t)=\sqrt{x^{2}(t)+y^{2}(t)} \\
\theta(t)=2 k \pi+\theta_{1}(t) \\
\theta\left(t_{2}\right)>\theta\left(t_{1}\right), \text { if } t_{2}>t_{1}
\end{array}\right.
$$

where $k$ denote integer numbers and $\theta(t)$ denotes monotonically increasing continuous function; $A(t)$ and $\theta(t)$ can be considered instantaneous amplitudes and phases of $c(t)$, respectively; $\theta_{1}(t)$ can be considered as principal instantaneous phases of $c(t)$ given by

$$
\theta_{1}(t)=\left\{\begin{array}{l}
\theta_{0}(t), \text { if } x(t)>0, y(t) \geq 0 \\
0.5 \pi, \text { if } x(t)=0, y(t) \geq 0 \\
\pi+\theta_{0}(t), \text { if } x(t)<0, y(t) \geq 0 \\
\pi+\theta_{0}(t), \text { if } x(t)<0, y(t)<0 \\
1.5 \pi, \text { if } x(t)=0, y(t)<0 \\
2 \pi+\theta_{0}(t), \text { if } x(t)>0, y(t)<0
\end{array}\right.
$$

and

$$
\theta_{0}(t)=\arctan \left[x^{-1}(t) \cdot y(t)\right] \in(-0.5 \pi, 0.5 \pi)
$$

where $\arctan []$ denotes arctangent function. 
As principal periods (or period-jumps) of $\theta_{0}(t)$ and $\theta_{1}(t)$ are $\pi$ and $2 \pi$, respectively; thus, $\theta_{0}(t)$ and $\theta_{1}(t)$ are both discontinuous functions, and their derivatives do not exist. Therefore, instantaneous frequencies of $c(t)$ can be given by $[22,23]$

$$
v(t)=\frac{1}{2 \pi} \frac{\partial \theta(t)}{\partial t}
$$

If taking natural logarithm to Equation (A2), we have

$$
i \theta(t)=\ln [c(t)]-\ln [A(t)] .
$$

Then, Equation (A6) can be expressed as

$$
v(t)=\operatorname{Re}\left[\frac{1}{2 \pi i} \frac{1}{c(t)} \frac{\partial c(t)}{\partial t}\right]=\frac{1}{2 \pi} \frac{x(t) \frac{\partial y(t)}{\partial t}-y(t) \frac{\partial x(t)}{\partial t}}{x^{2}(t)+y^{2}(t)} .
$$

Usually, $x(t)$ and $y(t)$ are both implicit, and we cannot directly obtain their derivatives; but, if Fourier transform [22] results of $c(t), x(t)$, and $y(t)$ all exist, we can use them to obtain the corresponding derivatives; it means that if

$$
\left\{\begin{array}{l}
0 \leq \int_{-\infty}^{+\infty} x^{2}(t) d t<+\infty \\
0 \leq \int_{-\infty}^{+\infty} y^{2}(t) d t<+\infty
\end{array}\right.
$$

then

and

$$
\left\{\begin{array}{l}
\frac{\partial c(t)}{\partial t}=c_{0}(t) \\
\frac{\partial x(t)}{\partial t}=x_{0}(t)=\operatorname{Re}\left[c_{1}(t)\right] \\
\frac{\partial y(t)}{\partial t}=y_{0}(t)=\operatorname{Re}\left[c_{2}(t)\right]
\end{array}\right.
$$

$$
\left\{\begin{array}{l}
c_{0}(t)=\int[i 2 \pi \eta \cdot C(\eta)] \cdot \exp [i 2 \pi \eta t] d \eta \\
c_{1}(t)=\int[i 2 \pi \eta \cdot X(\eta)] \cdot \exp [i 2 \pi \eta t] d \eta \\
c_{2}(t)=\int[i 2 \pi \eta \cdot Y(\eta)] \cdot \exp [i 2 \pi \eta t] d \eta
\end{array}\right.
$$

where $\operatorname{Re}[]$ takes the real part of a complex number; $c_{0}(t), c_{1}(t)$, and $c_{2}(t)$ are derivatives of $c(t), x(t)$, and $y(t)$, respectively; $\eta$ is the frequency; $C(\eta), X(\eta)$, and $Y(\eta)$ denote the Fourier transform results of $c(t), x(t)$, and $y(t)$, respectively; thus, Equation (A8) can be rewritten as

$$
v(t)=\operatorname{Re}\left[\frac{1}{2 \pi i} \frac{c_{0}(t)}{c(t)}\right]=\frac{1}{2 \pi} \frac{x(t) \cdot y_{0}(t)-y(t) \cdot x_{0}(t)}{x^{2}(t)+y^{2}(t)} .
$$

Due to impacts of dynamic range $[9,21]$ and amplitude amplified scale factor $2 \pi \eta$, background noises beyond effective bandwidths $[9,21]$ of $C(\eta), X(\eta)$, and $Y(\eta)$ will seriously affect the stabilities of $c_{0}(t), c_{1}(t)$, and $c_{2}(t)$, respectively, and finally reduce the stabilities and precisions of $v(t)$. Usually, we can replace derivatives $c_{0}(t), c_{1}(t)$, and $c_{2}(t)$ in Equation (A12) with their corresponding differences; thus, estimated instantaneous frequencies can be expressed as

$$
v_{1}(t)=\operatorname{Re}\left[\frac{1}{2 \pi i} \frac{d_{0}(t)}{c(t)}\right]=\frac{1}{2 \pi} \frac{x(t) \cdot d_{2}(t)-y(t) \cdot d_{1}(t)}{x^{2}(t)+y^{2}(t)}
$$

and

$$
\left\{\begin{array}{l}
d_{0}(t)=\frac{c(t)-c(t-\Delta t)}{\Delta t} \\
d_{1}(t)=\frac{x(t)-x(t-\Delta t)}{\Delta t} \\
d_{2}(t)=\frac{y(t)-y(t-\Delta t)}{\Delta t}
\end{array}\right.
$$

where $\Delta t$ denotes the time-sample interval. 
If $x(t)$ and $y(t)$ do not meet the requirements expressed in Equation (A9), such as $x(t)=t^{2}$ and so on, then $x(t)$ and $y(t)$ do not have Fourier transform results; therefore, we cannot obtain derivatives $c_{0}(t), c_{1}(t)$, and $c_{2}(t)$, but we can replace them in Equation (A12) with their corresponding differences. Correspondingly, the estimated instantaneous frequencies can be expressed as Equations (A13) and (A14).

Above all, the estimated instantaneous frequencies can be expressed as

$$
v_{1}(t)=\operatorname{Re}\left[\frac{1}{2 \pi \Delta t} \frac{c(t)-c(t-\Delta t)}{i \cdot c(t)}\right]=\frac{1}{2 \pi \Delta t} \frac{x(t-\Delta t) y(t)-x(t) y(t-\Delta t)}{x^{2}(t)+y^{2}(t)}
$$

which is considered the conventional instantaneous frequencies estimation method (CIF). Equation (A15) shows that the estimated instantaneous frequencies are affected by the timesample interval, and precisions of estimated instantaneous frequencies are decreased with the increasing time-sample interval [21]; thus, CIF may lead to inaccurate instantaneous frequency estimation results.

\section{Appendix B. High-Precision Instantaneous Frequencies Estimation Methods via Principal Instantaneous Phases}

Equation (A6) can be rewritten as

$$
v(t)=\frac{1}{2 \pi} \frac{\partial \theta(t)}{\partial t}=\frac{1}{2 \pi} \lim _{\Delta t \rightarrow 0}\left[\frac{\theta(t)-\theta(t-\Delta t)}{\Delta t}\right]
$$

as parameter $k$ in Equation (A3) cannot be determined, thus, $\theta(t)$ cannot be directly used for instantaneous frequencies estimation; but if we can avoid period-jumps of $\theta_{0}(t)$ and $\theta_{1}(t)$, then they can be both directly used for instantaneous frequencies estimation.

If $\theta_{0}(t)$ is used for instantaneous frequencies estimation, then according to Equation (A16), the estimated instantaneous frequencies can be obtained by computing the derivative of arctangent function itself as [23]

$$
p_{1}(t)=\frac{1}{2 \pi} \frac{\partial \theta_{0}(t)}{\partial t}=\frac{1}{2 \pi} \frac{\partial \arctan \left[x^{-1}(t) \cdot y(t)\right]}{\partial t}
$$

and

$$
p_{1}(t)=\frac{1}{2 \pi} \frac{x(t) \frac{\partial y(t)}{\partial t}-y(t) \frac{\partial x(t)}{\partial t}}{x^{2}(t)+y^{2}(t)}=v(t) .
$$

Therefore, according to Equations (A9)-(A14), the estimated instantaneous frequencies can be expressed as

$$
p_{2}(t)=\frac{1}{2 \pi \Delta t} \frac{x(t-\Delta t) y(t)-x(t) y(t-\Delta t)}{x^{2}(t)+y^{2}(t)}=v_{1}(t),
$$

and it means that this method can avoid phase period-jumps, but it cannot avoid the impact of the time-sample interval and may lead to inaccurate instantaneous frequencies estimation results.

If $\theta_{1}(t)$ is used for instantaneous frequencies estimation, then according to Equation (A16), the estimated instantaneous frequencies can be given by

$$
p_{3}(t)=\frac{1}{2 \pi} \frac{\theta_{1}(t)-\theta_{1}(t-\Delta t)}{\Delta t}
$$

and it avoids the impact of the time-sample interval to obtain high-precision instantaneous frequencies, except for time-points corresponding to period-jumps of $\theta_{1}(t)$.

If we consider

$$
\Delta \theta_{1}(t)=\theta_{1}(t)-\theta_{1}(t-\Delta t)
$$


then

$$
-2 \pi \leq \Delta \theta_{1}(t) \leq 2 \pi
$$

Based on Equation (A21), if we consider

$$
\Delta \theta(t)=\theta(t)-\theta(t-\Delta t)=2 \pi \times \Delta t \times v(t),
$$

then according to the sampling theorem $[26,27]$, there should be

$$
-\pi \leq \Delta \theta(t) \leq \pi .
$$

Thus, we can modify $\Delta \theta_{1}(t)$ into $\Delta \theta_{2}(t)$ to meet the requirement expressed in Equation (A24) as

$$
\Delta \theta_{2}(t)=\left\{\begin{array}{l}
-\Delta \theta_{1}(t)-2 \pi, \text { if } \quad-2 \pi \leq \Delta \theta_{1}(t)<-\pi \\
\Delta \theta_{1}(t), \text { if }-\pi \leq \Delta \theta_{1}(t) \leq \pi \\
\Delta \theta_{1}(t)-2 \pi, \quad \text { if } \pi<\Delta \theta_{1}(t) \leq 2 \pi
\end{array} .\right.
$$

Then

$$
-\pi \leq \Delta \theta_{2}(t) \leq \pi
$$

and estimated high-precision instantaneous frequencies can be given by

$$
h_{1}(t)=\frac{1}{2 \pi} \frac{\Delta \theta_{2}(t)}{\Delta t}
$$

and it avoids impacts of time-sample interval and period-jumps of $\theta_{1}(t)$.

\section{References}

1. Gabor, D. Theory of communication. J. Inst. Electr. Eng. 1946, 93, 429-457. [CrossRef]

2. Kronland-Martinet, R.; Morlet, J.; Grossmann, A. Analysis of sound patterns through wavelet transforms. Int. J. Pattern Recognit. Artif. Intell. 1987, 1, 273-302. [CrossRef]

3. Daubechies, I.; Maes, S. A nonlinear squeezing of the continuous wavelet transform based on auditory nerve models. In Wavelets in Medicine and Biology; CRC Press: Boca Raton, FL, USA, 1996; pp. 527-546.

4. Stockwell, R.G.; Mansinha, L.; Lowe, R.P. Localization of the complex spectrum: The S-transform. IEEE Trans. Signal Process. 1996, 44, 998-1001. [CrossRef]

5. Pinnegar, C.R.; Mansinha, L. The S-transform with windows of arbitrary and varying shape. Geophysics 2003, 68, 381-385. [CrossRef]

6. McFadden, P.D.; Cook, J.G.; Forster, L.M. Decomposition of gear vibration signals by the generalized S transform. Mech. Syst. Signal Process. 1999, 13, 691-707. [CrossRef]

7. Zhang, G.L. Time-phase amplitude spectra based on a modified short-time Fourier transform. Geophys. Prospect. 2018, 66, $34-46$. [CrossRef]

8. Zhang, G.L.; Li, Y.; Wang, T.Y.; Du, H.; Luo, F.; Zhan, Y.Z. Extended Hilbert transform and application for seismic attributes. Earth Space Sci. 2019, 6, 873-886. [CrossRef]

9. Li, Y.; Zhang, G.L.; Duan, J.; He, C.J.; Du, H.; Luo, F.; Zhan, Y.Z.; Wang, J. Extended stable factor method for the inverse Q-filter. Geophysics 2020, 85, T155-T163. [CrossRef]

10. Daubechies, I.; Lu, J.F.; Wu, H.T. Synchrosqueezed wavelet transforms: An empirical mode decomposition-like tool. Appl. Comput. Harmon. Anal. 2011, 30, 243-261. [CrossRef]

11. Meignen, S.; Oberlin, T.; McLaughlin, S. A new algorithm for multicomponent signals analysis based on synchrosqueezing: With an application to signal sampling and denoising. IEEE Trans. Signal Process. 2012, 60, 5787-5798. [CrossRef]

12. Auger, F.; Flandrin, P.; Lin, Y.T.; McLaughlin, S.; Meignen, S.; Oberlin, T.; Wu, H.T. Time-frequency reassignment and synchrosqueezing: An overview. IEEE Signal Process. Mag. 2013, 30, 32-41. [CrossRef]

13. Herrera, R.H.; Han, J.J.; Baan, M.V.D. Applications of the synchrosqueezing transform in seismic time-frequency analysis. Geophysics 2014, 79, V55-V64. [CrossRef]

14. Wang, P.; Gao, J.; Wang, Z. Time-frequency analysis of seismic data using synchrosqueezing transform. IEEE Geosci. Remote Sens. Lett. 2014, 11, 2042-2044. [CrossRef]

15. Oberlin, T.; Meignen, S.; Perrier, V. Second-order synchrosqueezing transform or invertible reassignment? Towards ideal time-frequency representations. IEEE Trans. Signal Process. 2015, 63, 1335-1344. [CrossRef]

16. Huang, Z.L.; Zhang, J.Z.; Zhao, T.H.; Sun, Y.B. Synchrosqueezing S-transform and its application in seismic spectral decomposition. IEEE Trans. Geosci. Remote Sens. 2015, 54, 817-825. [CrossRef] 
17. Ahrabian, A.; Looney, D.; Stanković, L.; Mandic, D.P. Synchrosqueezing-based time-frequency analysis of multivariate data. Signal Process. 2015, 106, 331-341. [CrossRef]

18. Huang, Z.L.; Zhang, J.Z.; Zou, Z.H. A second-order synchrosqueezing S-transform and its application in seismic spectral decomposition. Chin. J. Geophys. 2017, 60, 2833-2844.

19. Pham, D.H.; Meignen, S. High-order synchrosqueezing transform for multicomponent signals analysis-with an application to gravitational-wave signal. IEEE Trans. Signal Process. 2017, 65, 3168-3178. [CrossRef]

20. Xue, Y.J.; Cao, J.X.; Zhang, G.L.; Cheng, G.H.; Chen, H. Application of synchrosqueezed wavelet transforms to estimate the reservoir fluid mobility. Geophys. Prospecting. 2018, 66, 1358-1371. [CrossRef]

21. Zhang, G.L.; Duan, J.; Li, Y.; He, C.J.; Zhan, Y.Z.; Wang, J. Adaptive time-resample high-resolution synchrosqueezing transform and its application in seismic data. IEEE Trans. Geosci. Remote Sens. 2020, 58, 6691-6698. [CrossRef]

22. Bracewell, R.N. The Fourier Transform and Its Applications; McGraw-Hill Book Co. Inc.: New York, NY, USA, $1965 ;$ pp. $268-271$.

23. Taner, M.T.; Koehler, F.; Sheriff, R.E. Complex seismic trace analysis. Geophysics 1979, 44, 1041-1063. [CrossRef]

24. Li, B.; Xu, N.W.; Dai, F.; Zhang, G.L.; Xiao, P.W. Dynamic analysis of rock mass deformation in large underground caverns considering microseismic data. Int. J. Rock Mech. Min. Sci. 2019, 122, 104078. [CrossRef]

25. Li, B.; Xu, N.W.; Dai, F.; Gu, G.K.; Ke, W. Microseismic monitoring and stability analysis for the large-scale underground caverns at the Wudongde hydropower station. Bull. Eng. Geol. Environ. 2020, 79, 3559-3573. [CrossRef]

26. Nyquist, H. Certain topics in telegraph transmission theory. Trans. Am. Inst. Electr. Eng. 1928, 47, 617-644. [CrossRef]

27. Shannon, C.E. Communication in the presence of noise. Proc. IRE. 1949, 37, 10-21. [CrossRef] 